\title{
Genetic information leaflets: Influencing attitudes towards genetic testing
}

\author{
Susan Michie, DPhil, CPsychol ${ }^{1}$, Elena di Lorenzo, BSc ${ }^{1}$, Ruth Lane, BSc ${ }^{1}$, Kevin Armstrong, BSc ${ }^{1}$, and
} Saskia Sanderson, $B S c^{2}$

\begin{abstract}
Purpose: This article explores how a "neutral" genetics information leaflet influenced people's attitudes to be more positive toward predictive genetic testing. This is of concern, given the desire within clinical genetics and population based testing to provide information that informs choice without directing toward, or against, testing. Method: Four studies are reported. The first two investigated presentation (glossy and colored vs. black and white), and method of reading (read only vs. read followed by probing questions). The second two investigated content, using "think aloud," "card sort," and delayed recall tasks. Results: Those receiving a glossy leaflet expressed more positive attitudes and more interest in undergoing testing than those receiving a black and white leaflet, and those who were asked questions about what they had read were more positive about genetic testing than those who only read the leaflet. Recall one week later varied from $72 \%$ to $28 \%$, depending on type of information. Information that described the advantages of genetic testing or discussed genes and genetic testing in relation to disease were well recalled and rated positively. Attitudes toward information ranged from $100 \%$ positive (e.g., what diseases genetic tests are available for) to $0 \%$ positive (e.g., the meaning of a positive result). Conclusion: These results show that quite small changes within a leaflet can change attitudes toward genetic testing. This is of concern, given the association between attitudes toward a behavior and undergoing that behavior. The form, method of presentation, and content of genetic information leaflets should be evaluated for impact on attitude and decisions before they are used clinically. Genet Med 2004:6(4):219-225.
\end{abstract}

Key Words: information leaflets, genetic communication, attitudes, nondirectiveness, informed choice

Good clinical practice is to give those offered genetic testing both written and verbal information before testing to help them make an informed choice about whether or not to undergo testing. ${ }^{1,2}$ Written information is desirable, not only because it helps to inform the persons present at the consultation, it also helps to inform family members who were not present, but who may take part in making decisions. There is a strongly held professional consensus in the international genetics community that such information should be "neutral" or "nondirective" (as evident in textbooks over the last 50 years ${ }^{3,4}$ ). "Nondirective" communication in this context means that information is given in a way that does not influence people's attitudes toward, or decisions about, undergoing genetic testing. Despite this rhetoric, it has been found that information given during genetic counseling is not always nondirective. ${ }^{5}$

\footnotetext{
From the ${ }^{1}$ Department of Psychology and ${ }^{2}$ Epidemiology and Public Health, University College London, UK.

Copies of the leaflets are available via the ArticlePlus feature at the Genetics in Medicine Web site, http://www.geneticsinmedicine.org

Dr Susan Michie, MPhil, DPhil, CPsychol, FBPsS, Department of Psychology, University College London, 1-19 Torrington Place, London WC1E 7 HB.

Received: December 23, 2004.

Accepted: April 21, 2004.
}

DOI: 10.1097/01.GIM.0000132685.60259.EA
Increasingly, there is a demand for written health information to be provided to patients to empower them to make informed choices and to manage their own health. ${ }^{1,6}$ However, there is a concern that the quality of such leaflets is seldom evaluated. ${ }^{7}$ Given the sensitivity about providing genetic information in a nondirective way, it is important that patient information leaflets be evaluated for their impact on readers' attitudes and intention toward genetic testing.

The effects of a genetic information leaflet on attitudes toward genetic testing was recently examined in a study by Sanderson and colleagues (unpublished data, 2002). A simple, basic information leaflet about genetics and genetic testing was developed, based on internet-based genetics information resources (http://familydoctor.org/handouts/462. html and http://press2.nci.nih.gov/sciencebehind/genetesting/ genetesting01.htm). The leaflet (available from first author) was double-sided, A5, color and glossy, with subheadings "The Genetics and Health Survey" and "University of London." It provided information under the following headings: "What are genes?," "How are genes related to disease?," "What is genetic testing?," "What does a positive result mean?," "What does a negative result mean?," and "What are the advantages of being tested?" The leaflet was piloted with 71 individuals and a focus group, with the final version approved by a UK genetics specialist. 
In the survey of 1024 participants randomized to either receive or not receive this leaflet, it was found that receiving the leaflet was associated with more positive attitudes to genetic testing (unpublished data, 2002). Attitude was measured by asking respondents to check one or more of 12 positive, ambivalent, or negative words that described how they felt about genetic testing. ${ }^{8} 41 \%$ of the 508 receiving the leaflet checked positive or ambivalent words, compared to $28 \%$ of the 405 not receiving the leaflet, and $19 \%$ checked negative words, compared to $27 \%$ not receiving the leaflet (chi-square $=21.88, P<$ 0.001). It appears that some aspect of either the leaflet's content or appearance led to positive attitudes, despite the leaflet having been designed to present information in a neutral manner. Given the association between attitudes toward a behavior and undergoing that behavior, ${ }^{9,10}$ it is important to understand more about the ways in which an information leaflet affects attitudes toward genetic testing, and interest in, and intention to undergo, genetic testing.

Studies of persuasion have suggested that information may influence attitudes and behavior through two cognitive processing routes: a central and a peripheral one. ${ }^{11}$ The central route requires a depth of information processing, and occurs when there is an opportunity to evaluate and integrate information whereas the peripheral route uses contextual cues to make simple inferences about the merits of the information content without using complex cognitive processing. ${ }^{12}$ Examples of contextual cues are attractiveness of presentation and source credibility. Persuasion that takes place more centrally has been found to lead to more stable attitudes that are more resistant to counter-persuasion and more predictive of behavior, than attitudes that have been influenced by more peripheral information processing.

\section{MATERIALS AND METHODS}

This article reports four studies aimed at investigating whether this genetic information leaflet influences attitude via peripheral or central information processing (studies 1 and 2), and whether different parts of the leaflet have a differentially positive effect on attitudes (studies 3 and 4 ). The use of peripheral cues was assessed by comparing the impact of attractive leaflets from a credible source with the impact of less attractive, unsourced leaflets. The former is predicted to lead to more positive attitudes. Central processing was assessed by comparing the impact of the leaflet on those who have been questioned about issues related to its content after reading it with those who have only read them. The former facilitates deeper processing, predicted to be associated with positive attitudes toward testing. The impact of the leaflet was assessed by questionnaire measures of attitudes toward genetic testing, interest in undergoing a genetic test, and intention to undergo such a test if offered one.

The third study used "think aloud" and "card sort" tasks and the fourth used a delayed recall technique. The first task involved people verbalizing their thoughts as they read the leaflet. Those sections generating more positive thoughts are likely to be those influencing attitude in a positive direction. The second task reproduced each statement from the leaflet on separate cards. People were asked to sort the statements according to whether or not they had been influenced by them, and whether this was in a positive or negative direction. Those statements judged to have had a positive impact were hypothesized to be those that created a positive attitude. In the final study, recall of information one week after reading the leaflet was assessed by a series of questions relating to different sections of the leaflet. The parts of the leaflet that were best recalled were assumed to be the most salient and, therefore, the most likely to influence attitudes. Ethical approval for the studies was granted by the University College London/University College London Hospitals Joint Research Ethics Committee (ref: 02/0272). Potential participants were invited to take part in a research study looking at the impact of health information leaflets were given a study information sheet to read and gave written consent to study participation.

\section{Study 1: Impact of leaflet appearance}

\section{Hypothesis}

Reading glossy, sourced leaflets is associated with more positive attitudes, interest, and intention to undergo genetic testing than reading black and white, unsourced leaflets.

\section{Design}

An experimental, between-subjects design, with leaflet type as the independent variable. Leaflets included the same information, but were of two types: color, glossy, in brochure format and sourced as University College London, or black and white, on A4 sheets, with no picture and no source mentioned. The two types were distributed to participants in sequential blocks of 10 .

\section{Sample}

One hundred participants were recruited opportunistically, mainly among London University students. Response rate was $100 \%$. The study was powered to detect a medium effect size. There were no differences between experimental group on any of the demographic variables (Table 1).

\section{Measures}

(1) Socioeconomic status was assessed by education, home ownership, and access to car. Respondents were also asked about age, gender, and ethnic background.

(2) Attitudes toward genetic testing in general were assessed by a word checklist, ${ }^{13}$ comprising positive words (enthusiastic, optimistic, hopeful, and excited), ambivalent words (indifferent, cautious, confused, and mixed feelings), and negative words (horrified, concerned, pessimistic, and worried). Respondents were assigned to two categories, those checking positive or positive + ambivalent words, and those checking negative, ambivalent, or a combination of words that included at least one negative word. Participants were also asked to rate their attitude to undergoing a genetic test on 5-point scales: "a 
Table 1

Demographic characteristics of participants

\begin{tabular}{|c|c|c|c|c|c|c|}
\hline & \multicolumn{3}{|c|}{ Study 1} & \multicolumn{3}{|c|}{ Study 2} \\
\hline & Glossy & Black/White & Difference & Read only & Probes & Difference \\
\hline \multicolumn{7}{|l|}{ Gender } \\
\hline Male & 23 & 27 & \multirow{3}{*}{ NS } & 12 & 17 & \multirow{3}{*}{ NS } \\
\hline Female & 26 & 20 & & 18 & 13 & \\
\hline Missing & 1 & 3 & & 0 & 0 & \\
\hline Age (years) & $31(15.5)$ & $28(12.8)$ & NS & $23(9.8)$ & $25(2.6)$ & NS \\
\hline \multicolumn{7}{|l|}{ Living arrangements } \\
\hline Don't own home & 33 & 33 & \multirow{3}{*}{ NS } & 19 & 23 & \multirow{3}{*}{ NS } \\
\hline Own home & 16 & 14 & & 11 & 7 & \\
\hline Missing & 1 & 3 & & 0 & 0 & \\
\hline \multicolumn{7}{|l|}{ Car } \\
\hline No & 18 & 23 & \multirow{3}{*}{ NS } & 9 & 9 & \multirow{3}{*}{ NS } \\
\hline Yes & 31 & 24 & & 21 & 21 & \\
\hline Missing & 1 & 3 & & 0 & 0 & \\
\hline \multicolumn{7}{|c|}{ Highest educational qualification } \\
\hline School/vocational/other & 16 & 19 & \multirow{3}{*}{ NS } & 1 & 2 & \multirow{3}{*}{ NS } \\
\hline University degree & 33 & 28 & & 29 & 28 & \\
\hline Missing/other & 1 & 3 & & 0 & 0 & \\
\hline \multicolumn{7}{|l|}{ Ethnic Group } \\
\hline White & 39 & 33 & & 22 & 23 & \multirow{3}{*}{ NS } \\
\hline Non-White & 10 & 12 & NS & 8 & 7 & \\
\hline Missing & 1 & 5 & & 0 & 0 & \\
\hline
\end{tabular}

$\mathrm{n}$ and mean $(\mathrm{SD})$.

bad thing-a good thing," "beneficial-harmful," "importantunimportant." The alpha coefficient of reliability was 0.79 in studies 1 and 2, and the correlations with the categorical measure were 0.39 for study 1 and 0.34 for Study 2, suggesting that the personalized attitude scale is measuring an overlapping, but different, construct than the more generalized categorical measure.

(3) Interest in undergoing a genetic test was assessed by a scale formed by summing the responses to the following four items: (1) "Suppose you inherited something from your parents which made you more likely to develop cancer than other people; would you want to be told this?," (2) "Would you be interested in taking a genetic test for cancer risk?," (3) "Would you have a genetic test for cancer risk if your doctor recommended it?," and (4) "If it were available now, would you have a genetic test for cancer risk in the next 6 months?" Response options were "no, definitely not"; "no, probably not"; "yes, probably"; and "yes, definitely," with an alpha coefficient of reliability $>0.80$ for studies 1 and 2 .

(4) Intention to undergo a genetic test was measured by the item "If offered such a test:" with five possible response options, ranging from "I would definitely not intend to undergo it," to "I would definitely intend to undergo it."

\section{Procedure}

Participants were given the leaflet to read and then asked to complete a brief questionnaire.

\section{Analysis}

Differences between groups were analyzed by independent group $t$ tests and by chi-square tests. Because the hypotheses were directional, one-sided tests were used, giving a significance level of $P=0.10$.

Results

As predicted, those receiving the glossy leaflets had more positive attitudes toward genetic testing on both the general checklist measure and the more personal rating scale measure. They were also more interested in undergoing genetic testing and expressed greater intention to undergo testing if they received the glossy leaflet (Table 2).

\section{Study 2: Impact of encouraging deeper information processing}

\section{Hypothesis}

Answering probing questions after the reading of the genetic information leaflet (the glossy, colored, sourced version) is associated with more positive attitudes, interest, and intention to undergo testing than reading the leaflet without questions.

\section{Design}

An experimental, between-subjects design with presence or absence of probing questions about the leaflet information as the independent variable.

\section{Sample}

Sixty London University undergraduates were recruited opportunistically, 30 for the read-only group and 30 for the probe group. Response rate was 60\% (30/50) for the read only group (which completed the task at the beginning of a lecture) and $100 \%$ for the probe group who were invited to take part individually. The study was powered to detect a medium effect size. 
Table 2

Attitudes, interest, and intention in those receiving glossy and black and white leaflets ${ }^{a}$

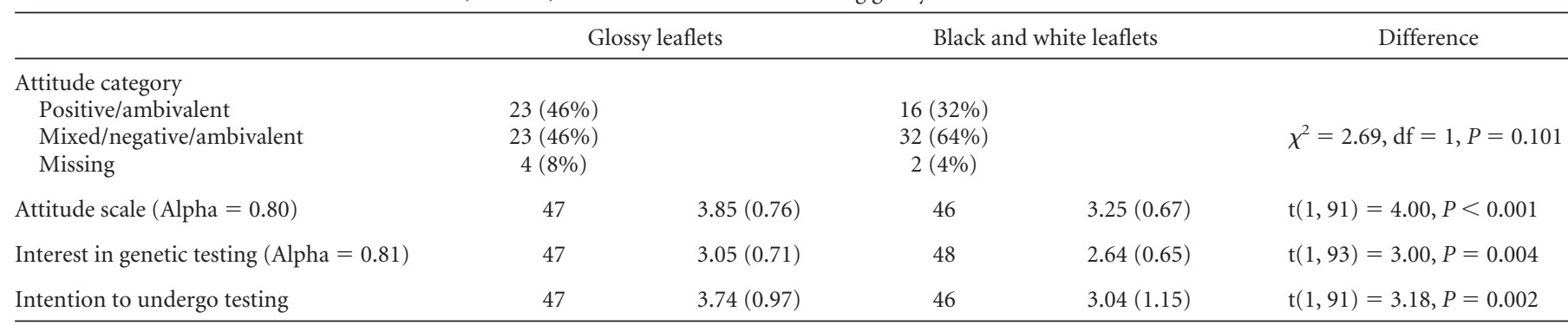

${ }^{a}$ There are between two and four missing data points within each group for the three scales.

There were no differences between experimental group on any of the demographic variables (Table 1).

\section{Measures}

Measures of demographic characteristics and of leaflet impact were the same as those used for Study 1.

\section{Procedure}

Participants were asked to read the genetic information leaflet and to complete a questionnaire. The setting was a lecture theater for the "read only" group and a University office for the "probe" group. The "probe" group was given the following written questions to answer in writing before completing the questionnaire: (1) What do you know about genes?, (2) What do you know about genetic tests?, (3) What can genetic tests tell you?, and (4) What are your views about genetic testing?

\section{Analysis}

Differences between groups were analyzed by independent group $t$ tests and by chi-square tests.

\section{Results}

As predicted, those who were asked questions that probed the material read in the leaflet held more positive attitudes toward genetic testing, as measured by the word checklist (Table 3). However, there were no differences between groups in the more personally orientated attitude, interest, and intention scales.

\section{Study 3: Think aloud and card sort tasks}

Aim

To investigate which parts of the genetic information leaflet were evaluated most positively.

\section{Design}

This study was a within-subjects design, with all participants completing the think aloud task before the card sort task. The tasks were in this order because the card sort imposes a structure on participants' responses, which may bias subsequent response in the think-aloud task.

\section{Sample}

Fifteen of 16 people recruited from a leisure center, a primary school, and a university completed the practice task satisfactorily and participated in the study. Nine were women and the age range was 19 to 45 years, with a mean of 27 years (SD 8.5 years). Ten described themselves as white, three as Asian, and two as African.

\section{Procedure}

(1) Think aloud task: Concurrent rather than retrospective verbal reporting was used, because a delay between completing the task and verbally reporting can introduce "error." 14 In concurrent reporting, participants verbalize their thoughts in the pause immediately after reading each meaningful text segment, facilitating the expression of thoughts in short-term

Table 3

Attitudes, interest, and intention in those without and with probes

\begin{tabular}{|c|c|c|c|c|c|c|c|}
\hline & \multicolumn{3}{|c|}{ Read only } & \multicolumn{3}{|c|}{ Probe } & \multirow{2}{*}{$\begin{array}{c}\text { Difference } \\
\mathrm{t}(1,58)=6.00, P<0.001\end{array}$} \\
\hline Total items recalled (mean and SD, $\max 29$ ) & \multicolumn{2}{|c|}{$9.53(2.38)$} & & \multicolumn{2}{|c|}{$12.83(1.84)$} & & \\
\hline \multicolumn{8}{|l|}{ Attitude category } \\
\hline Positive/ambivalent & 12 & $(35 \%)$ & & 22 & $(65 \%)$ & & \\
\hline Mixed/negative/ambivalent & 18 & $(59 \%)$ & & 8 & $(31 \%)$ & & $\chi^{2}=6.79, \mathrm{df}=1, P<0.009$ \\
\hline Attitude scale (Alpha $=0.79)$ & & 30 & $3.56(0.63)$ & & 30 & $3.48(0.67)$ & $\mathrm{t}(1,58)=0.46, \mathrm{NS}$ \\
\hline Interest in genetic testing $($ Alpha $=0.86)$ & & 30 & $2.96(0.62)$ & & 30 & $2.75(0.68)$ & $\mathrm{t}(1,58)=1.24, \mathrm{NS}$ \\
\hline Intention to undergo testing & & 30 & $3.67(0.88)$ & & 30 & $3.37(1.03)$ & $\mathrm{t}(1,58)=1.21, \mathrm{NS}$ \\
\hline
\end{tabular}


memory. ${ }^{15}$ Participants were asked to read the leaflet out loud and to verbalize everything they were thinking and not to plan what they were saying or to explain what they were thinking. ${ }^{16}$ The session was audio tape recorded.

(2) Card sort task: Participants were presented with 41 cards, each showing one of the leaflet segments used in the think aloud task, and were asked to sort the cards into two piles, according to whether or not the statements had had an impact on them. They then sorted those that had had an impact into one of five categories: "very positive," "quite positive," "neutral," "quite negative," or "very negative" about genetic testing.

\section{Analysis}

Sentence structure and content were used to help the segmentation of the verbatim ${ }^{16}$ into statements, each representing one main idea (these were the same as used in the card sort task). The interrater reliability was $97.1 \%$. Each statement was coded as "very positive" $(+2)$, "quite positive" $(+1)$, "neutral" $(0)$, "quite negative" $(-1)$, or "very negative" $(-2)$ about genetic testing. The interrater reliability for the think aloud task procedure was 0.86 , indicating a high level agreement. ${ }^{17}$ An average, score was calculated to give a mean score for each statement. For the card sort task, the average score for each statement was the total score across participants divided by the number of participants that had singled out that statement as having had an "impact."

\section{Results}

There was a positive association between the number of statements rated as positive, neutral, and negative in the two tasks $(r=0.64, P<0.01$; Table 4$)$. Statements rated positively in both tasks were those that gave information about genes, mentioned future developments, provided reassurance about risk, or mentioned usefulness to the family. Statements rated negatively in both tasks were those that mentioned things that might cause worry, described limitations of testing, pointed out the uncertainty of results, or mentioned the blood test.

Of the 41 leaflet statements, 24 were the substantive pieces of information used to assess recall in study 2 . The total numbers and percentages of pieces of information per leaflet section that were rated positively are shown in the first two columns of Table 5. These will be considered alongside the results of study 4.

\section{Study 4: Delayed recall task}

Aim

To investigate which parts of the genetic information leaflet are best recalled after one week.

\section{Design}

There were two independent groups, one in which people only read the leaflet and one in which people were asked probing questions after reading the leaflet.

\section{Sample}

The sample was the same as in study 2 .

\section{Recall measure}

Recall was measured by participants writing open-ended responses to eight questions, corresponding to the leaflet sections (see Appendix). Each question was associated with two, three, or four relevant pieces of information within the leaflet, giving a maximum recall score of 24 . Interrater agreement for judgment of correct responses was $87 \%$. The recall score was the mean percentage recall of each item for the sample, averaged across the two raters.

\section{Procedure}

Recall was assessed one week after reading the leaflet. The setting was a lecture theater for the "read-only" group and a University office for the "probe" group.

\section{Analysis}

The score for each question was converted to a percentage of the total marks available for that question. An independent samples $t$ test was performed on the total mean recall score of the leaflet to determine whether the "probes" groups recalled more than the "read only" group. A repeated measures ANOVA was conducted to determine which particular questions were better recalled by each group.

\section{Results}

Participants in the "probes" group recalled more information than those in the "read-only" group $[\mathrm{t}(58)=6.00, P<$ 0.001] (Table 5).

Certain leaflet sections were significantly better remembered than others, with a significant within-subjects effect

Table 4

Number of statements eliciting positive, neutral, and negative evaluations in both the think-aloud and card sort tasks

\begin{tabular}{|c|c|c|c|c|c|}
\hline & & \multicolumn{4}{|c|}{ Think-aloud } \\
\hline & & Positive & Neutral & Negative & Total \\
\hline \multirow{4}{*}{ Card sort } & Positive & 10 & 8 & 10 & $28(68.3 \%)$ \\
\hline & Neutral & 0 & 0 & 0 & $0(0 \%)$ \\
\hline & Negative & 0 & 3 & 10 & $13(31.7 \%)$ \\
\hline & Total & $10(24.4 \%)$ & $11(26.8 \%)$ & $20(48.8 \%)$ & 41 \\
\hline
\end{tabular}


Table 5

Numbers and percentages of pieces of information rated positively in the think aloud and card sort tasks, and the percentages of pieces of information recalled one week later

\begin{tabular}{|c|c|c|c|c|}
\hline \multirow{3}{*}{$\begin{array}{l}\text { Section of leaflet } \\
\text { How are genes related to disease? }\end{array}$} & \multirow{2}{*}{\multicolumn{2}{|c|}{$\begin{array}{l}\text { Number and \% pieces of } \\
\text { information rated positively } \\
\text { in both tasks }\end{array}$}} & \multicolumn{2}{|c|}{ Recall one week later } \\
\hline & & & \multirow{2}{*}{$\frac{\text { With probes }}{53 \%}$} & \multirow{2}{*}{$\frac{\text { Read only }}{39 \%^{\star *}}$} \\
\hline & $6 / 6$ & $100 \%$ & & \\
\hline What diseases are genetic tests currently available for? & $6 / 6$ & $100 \%$ & $52 \%$ & $40 \%$ \\
\hline What are the advantages of being tested? & $6 / 8$ & $75 \%$ & $53 \%$ & $40 \%{ }^{* *}$ \\
\hline What are genes? & $6 / 8$ & $75 \%$ & $28 \%$ & $24 \%$ \\
\hline What is genetic testing? & $2 / 4$ & $50 \%$ & $72 \%$ & $45 \% * * *$ \\
\hline What does a negative result mean? & $2 / 4$ & $50 \%$ & $40 \%$ & $29 \% *$ \\
\hline What are the disadvantages of being tested? & $0 / 8$ & $0 \%$ & $47 \%$ & $33 \%{ }^{* *}$ \\
\hline What does a positive result mean? & $0 / 4$ & $0 \%$ & $37 \%$ & $27 \% *$ \\
\hline
\end{tabular}

Difference between the "probes" and "read only" groups ${ }^{\star} P<0.05,{ }^{* *} P<0.01,{ }^{* * *} P<0.001$.

across section $[\mathrm{F}(7,406)=15.96, P<0.001]$. Recall was highest for items that described genetic testing, its links with disease, and the advantages of genetic testing. Recall was lowest for items that described the nature of genes, the meaning of test results, and the disadvantages of genetic testing.

The information that was both rated positively and recalled in more than half of the "probe" group was that describing how genes are related to disease, what diseases can be currently genetically tested for, and the advantages of being genetically tested. This information is likely to be most responsible for influencing attitudes toward genetic testing in a positive direction. Disadvantages of testing and the meaning of a positive result are rated negatively and recalled by a minority of people, suggesting little influence on positive attitudes.

\section{DISCUSSION}

These studies suggest that quite small differences in the way that a genetics information leaflet is written, presented, and discussed can influence attitude toward, interest in, and intention to undergo genetic testing and the extent to which information is recalled. They also suggest that the presentation and context of reading the leaflet influences attitudes in different ways. A glossy, colored, sourced leaflet with a picture led to more positive attitudes than a plain, black and white, unsourced, picture-less leaflet. Engaging people in an active process of answering questions about the content of the leaflet also led to more positive attitudes than merely asking them to read it. However, the form of presentation and questioning appeared to influence attitudes in different ways. Form of presentation influenced people's attitudes toward undergoing testing, but not toward genetic testing in general. On the other hand, asking questions, thus encouraging deeper processing, influenced attitudes toward genetic testing in general but not their attitudes, interest, or intention in relation to undergoing testing themselves. As discussed earlier, attitudes formed in this way are likely to be more stable and, therefore, more pre- dictive of behavior than attitudes shaped by more peripheral cues. Longitudinal studies are required to determine the stability and predictive power of attitudes toward genetic testing in general and toward undergoing testing oneself. A factorial study design that varied both form of presentation and presence of probing questions would allow the investigation of both main effects and interactions in influencing subsequent behavior. Studies are also needed to investigate the independent and synergistic effects of color, glossiness, source, and picture on attitudes, and potential mediators of the association between such variables and attitude.

The content of the leaflet influenced attitudes and recall in different, and not necessarily obvious, ways. For example, information about how genes are related to disease was universally perceived as positive, whereas information about genetic testing was seen as negative as frequently as it was seen as positive. On the other hand, information about genetic testing was recalled a week later more than twice as well as information about genes. In order to ascertain the influence that information has on attitudes toward undergoing testing and, hence, decisions about testing, both attitudes and recall need to be assessed. Future research should include a longer follow-up and ask participants to evaluate the information that they recalled.

The two techniques of judging the content of the leaflets gave broadly similar results. However, people were more likely to report negative statements in the "think aloud" than the "card sort" task. It may be that the card sort task introduced a social desirability bias, i.e., participants sorted cards in a way they believed to be socially acceptable and desirable. This difference, and the difference between methods of measuring attitude, highlights the need to include more than one type of measure of constructs being studied.

The studies reported in this article have used a variety of methods to investigate the impact of a genetics information leaflet on attitudes toward undergoing genetic testing. Two of the three datasets were drawn from students, and the need to 
replicate these results in clinical populations is evident. Despite this caveat, the results of these studies have implications for the objectives of providing "neutral" information about genetic testing. The impact of such information should be examined for population based testing as well as within the context of genetic consultations. However, criteria as to what constitutes neutral information are not clear. Some may argue that the goal of such a leaflet should be to achieve equal recall of positively and negatively rated information. Others may argue that this is imposing false neutrality on situations that may be viewed either positively or negatively. This begs the question as to who should judge how positive attitudes should be in different genetic testing situations.

These results point to the need to pilot information leaflets before use so that both health professionals and patients are aware of the likely impact of leaflets. The input of both groups into leaflet development will contribute to comprehensibility of the information and a "balanced" presentation of information. Decisions about what information to include, and how to present it, can then be taken on the basis of empirical data about its impact, rather than on the basis of personal views or past practice.

\section{Appendix}

The questions used to assess recall and the relevant pieces of information presented in the leaflet:

1 . What are genes?

- control the development and workings of your body

- made of DNA

- present in each cell of the body

- come in different versions called genetic variations

2. How are genes related to disease?

- genetic variations can sometimes lead to disease

- the gene, or a bit of it, may be abnormal or damaged

- some genetic variations occur in families and some occur by chance

3. What is genetic testing?

- tests look for genetic variations that may lead to disease

- help show likelihood of certain disease in the future

4. What does a positive result mean?

- individual has the variation they've been tested for

- means that one may be more likely to get the disease, but not that one will definitely get the disease

5 . What does a negative result mean?

- means that one does not have that particular variation
- one is not at a higher risk of contracting disease than other people, but does not mean that one won't ever get the disease

6. What are the advantages of being tested?

- not so worried about getting a disease

- can change lifestyle (diet, start exercising, or quit smoking to reduce risk)

- can take medicine

- result can provide family members with useful information about their own risk

7. What are the disadvantages of being tested?

- might become more worried about getting ill

- some illnesses can't be prevented

- could lead to problems with employers

- could affect life insurance

8. What diseases are genetic tests currently available for?

- cystic fibrosis

- some types of breast cancer

- bowel cancer

\section{References}

1. Holtzman NA, Watson MS. Promoting safe and effective genetic testing in the United States: Final report of the task force on genetic testing. National Institute of Health, 1997 Available at: http://www.genome.gov/10001733. Accessed April 20, 2004.

2. Department of Health. Our inheritance, our future: Realising the potential of genetics in the NHS. London: The Stationery Office, 2003.

3. Roberts JAF. An introduction to human genetics, 2nd ed. Oxford: Oxford University Press, 1959.

4. Skirton H, Patch C. Genetics for Healthcare Professionals. Oxford: BIOS; 2002:11.

5. Michie S, Bron F, Bobrow M, Marteau TM. Non-directiveness in genetic counseling: an empirical study. Am J Hum Genet 1997;60:40-47.

6. Department of Health. The NHS Plan: A plan for investment, a plan for reform. London: The Stationery Office; 2000.

7. Charnock D, Shepperd S, Needham G, Gann R. DISCERN: An instrument for judging the quality of written consumer health information on treatment choices. J Epidemiol Community Health 1993;53:105-111.

8. Michie S, Drake H, Bobrow M, Marteau T. A comparison of public and professionals' attitudes towards genetic developments. Public Underst Sci 1995;4:243-253.

9. Ajzen I. Nature and operation of attitudes. Annu Rev Psych 2001;52:27-58.

10. Chen M, Bargh JA. Consequences of automatic evaluation: immediate behavioural predispositions to approach or avoid the stimulus. Pers Soc Psych Bull 1999;22:215224.

11. Petty RE, Cacioppo JT. The elaboration likelihood model of persuasion. Adv Exp Soc Psych 1986;19:123-205.

12. Chaiken S, Wood W, Eagly AH. Principles of persuasion. In: Higgins ET, Kruglanski AW, editors. Social psychology handbook of basic principles. New York: Guilford, 1996:702-742.

13. Michie S, Drake H, Bobrow M, Marteau T. A comparison of public and professionals' attitudes towards genetic developments. Public Underst Sci 1995;4:243-253.

14. Green A. Verbal protocol analysis. Psychologist 1995;8:126-129.

15. Ericsson KA. Concurrent verbal reports on text comprehension: a review. Text 1988; 8:295-325.

16. Green C, Gilhooly K. Protocol analysis: practical implementation. In: Richardson JTE, editor. Handbook of qualitative research methods for psychology and social sciences. Leicester: BPS Books, 1996: 55-74.

17. Landis JR, Koch GG. The measurement of observer agreement for categorical data. Biometrics 1977;33:159-174. 